ARTÍCULO

\title{
Perspectiva de género en currículums educativos: obstáculos y avances en educación básica y media
}

\author{
Amanda Valenzuela-Valenzuelal (D) \\ Ricardo Cartes-Velásquez" (D)
}

RESUMEN

El sistema educativo es una de las instancias que permite la construcción de identidad de género del estudiantes masculinos y femeninos. Históricamente, el enfoque que se le ha dado al género en educación consiste en la desigualdad en el acceso de las estudiantes, pero esta no es la única problemática. En esta revisión, se abordan antecedentes del abordaje de género en educación, formas en que se ha trabajado el tema, la relevancia del currículum en la enseñanza de la perspectiva de género y los obstáculos en la incorporación de dicha perspectiva. Pese a la superación de múltiples barreras, los hallazgos permiten enunciar que debe trabajarse en la formación inicial y continua del profesorado, explicitando en el currículum las temáticas de género, y creando espacios de reflexión en las instituciones escolares.

\section{PALABRAS CLAVE}

currículum; enfoque de género; pedagogía en género. 


\title{
GENDER PERSPECTIVE IN EDUCATIONAL CURRICULA: OBSTACLES AND DEVELOPMENTS IN MIDDLE SCHOOL AND HIGH SCHOOL
}

\begin{abstract}
The educational system is one of the instances that allows the construction of the male and female students gender identity. Historically, the approach to gender in education has focused on inequality of access for female students, although this is not the only issue. This review addresses the background of gender approaches in education, the ways of tackling this issue, the relevance of the curriculum in teaching gender perspective and the obstacles to the inclusion of such perspective. Although multiple barriers have been surpassed, the findings support the claim that both initial and continuing teacher education must be improved, uncovering gender issues in the curriculum and creating opportunities for reflection in schools.
\end{abstract}

\section{KEYWORDS}

curriculum; gender approach; gender pedagogy.

\section{PERSPECTIVA DE GÊNERO NO CURRÍCULO EDUCACIONAL: OBSTÁCULOS E AVANÇOS NO ENSINO FUNDAMENTAL E MÉDIO}

\section{RESUMO}

O sistema educacional é uma das instâncias que permite a construção da identidade de gênero dos alunos e alunas. Historicamente, a abordagem de gênero na educação se restringiu à desigualdade de acesso para as alunas, mas esse não é o único problema. Nesta revisão, discutem-se os antecedentes da abordagem de gênero na educação, as maneiras pelas quais o assunto foi trabalhado, a relevância do currículo no ensino da perspectiva de gênero e os obstáculos à incorporação dessa perspectiva. Apesar da superação de múltiplas barreiras, os resultados permitem afirmar que é preciso trabalhar na formação inicial e continuada dos professores e professoras, explicitando questões de gênero no currículo e criando espaços de reflexão nas instituições escolares.

PALAVRAS-CHAVE

currículo; abordagem de gênero; educação não sexista. 


\section{INTRODUCCIÓN}

El concepto de género puede ser definido como las características femeninas o masculinas adquiridas por los individuos desde la cultura, a través de un proceso que es simultáneamente social e individual (Lamas, 1986); es un aprendizaje sociocultural, no definido por la biología, por lo que el género puede no coincidir con el sexo biológico de una persona, que se encuentra determinado por los cromosomas. De esta forma, los estudios de género se enfocan en cómo son adquiridas dichas características socioculturales por los individuos de una determinada cultura, viéndose el género como una categoría emergente que refiere a las relaciones entre mujeres y hombres, $\mathrm{y}$ a las construcciones sociales de masculinidad y femineidad. Esta categoría se basa en la construcción de un discurso de género por parte de la sociedad, en el cual hombres y mujeres son divididos en dos categorías opuestas, en las que niños y niñas se insertan desde una temprana edad, pudiendo mantener o resistirse a la categoría designada (Rodríguez y Peña, 2005).

Por su parte, la perspectiva de género se considera como lo vivido tanto por hombres como mujeres, y busca cambiar las expectativas tradicionales y estereotípicas de lo que es ser hombre y ser mujer, reconociendo la existencia de diferentes formas de ser hombres o mujeres (Gómez, 2009). La reflexión sobre las temáticas anteriormente mencionadas visibilizó las diferencias existentes entre ambos sexos como desigualdades sociales, económicas y políticas (Messina, 2001), desigualdades que llevan a que un grupo (el masculino y dominante) tenga ciertos privilegios por sobre otro (el femenino y dominado), lo cual ocurre por una construcción cultural, no por las capacidades naturales de cada género (Buquet, 2011; Gómez, 2009). Lo anterior corresponde a la desigualdad de género, en la cual Solís Pérez (2006) plantea que la mujer pasa a ser vista como inferior, limitando su participación en distintos ámbitos de la vida cotidiana, como lo son las esferas sociales, políticas y laborales. La autora añade que dicha desigualdad se debe a la "socialización diferenciada proveniente del hogar, de la escuela, la iglesia y los medios de comunicación” (Solís Pérez, 2006, p. 106), donde se refuerzan aquellos esquemas discriminatorios que son transmitidos por las distintas entidades.

Si bien el género es un elemento presente en todas las culturas desde los comienzos de la humanidad, el interés en estudiar la construcción del género al interior de instituciones educacionales es muy reciente, no surgiendo hasta la década de 1970 en la investigación educativa, gracias a un interés del feminismo de la época (Rodríguez y Peña, 2005). Este interés en estudiar el género desde la educación se debe a que esta corresponde a uno de los puntos principales de transmisión y transformación de patrones culturales de género (Colás y Jiménez, 2006), ya que es en los establecimientos educacionales donde las pautas y patrones culturales de una determinada sociedad son transmitidos y expresados, complementando las acciones de enseñanza familiares (Gómez, 2009). Por lo anterior, Rodríguez y Peña (2005) plantean que los establecimientos educacionales son el lugar ideal para iniciar los cambios de cualquier patrón cultural, en particular del 
género, puesto que se ven como instituciones eficaces de socialización secundaria, donde se les permite a niños y niñas la internalización de aquellas disposiciones básicas que se encuentran asociadas a cada género.

No obstante, pese a que los establecimientos educacionales pueden ser una instancia positiva para propiciar cambios, también son espacios en los cuales existen y se reproducen estereotipos y prácticas sexistas (Solís Pérez, 2006). Esto genera un trato diferenciado para niños y niñas de parte de los establecimientos de enseñanza básica y media. Durante la enseñanza, suele recalcarse la idea que las diferencias biológicas entre hombres y mujeres determinan sus roles en la vida social (Rodríguez y Peña, 2005), aun cuando, tal como se mencionó anteriormente, los roles de la vida social y la diferencia entre ambos géneros, dependen de la construcción social del género de cada individuo, no de lo determinado por la biología de cada persona. Se origina así, en el contexto escolar, un desbalance de poder entre hombres y mujeres, debido a los roles y valorizaciones que la cultura asigna a cada género (Viotti y Del Valle, 2013).

Los establecimientos educacionales, entonces, tienen influencia en sus estudiantes sobre cómo es vivido y construido el género; no son instituciones neutras en los procesos de construcción de relaciones e identidades de género (Madrid, 2006). Esto es logrado a través de la acción docente, puesto que, como plantea Blaise (2009), los profesores tienen el rol de formar el futuro de niños y niñas, por lo cual son una parte activa en la construcción de identidades de género de sus estudiantes. Añade, además, que niños y niñas son, también, agentes activos en la construcción de sus propias identidades género, al tener sus propias interpretaciones de qué es ser masculino o ser femenina, con la capacidad de tomar decisiones sobre el aceptar o resistir los discursos dominantes.

Actualmente, género y educación son un área de gran interés científico (Rebollo et al.,2011), pese a que las temáticas de género son casi inexistentes en la producción de material empírico (Madrid,2006), y pese a que aún no es un tema abordado del todo en el ámbito educativo, lo que conlleva a una falta de claridad y de herramientas de intervención (Gómez, 2009). Es en esto en lo que radica la importancia de investigar el género en la educación. Por ello, en el presente artículo, se realizará una revisión de la literatura internacional sobre el enfoque y la perspectiva de género en la enseñanza básica y media de niños y niñas, con un énfasis en por qué es imperante la incorporación de dicha perspectiva, con énfasis en los currículums escolares y las dificultades para su adecuación a esta perspectiva. Se presentarán antecedentes de la historia reciente que ha permitido el acercamiento de la perspectiva de género en el ámbito educativo en una primera sección, la cual será seguida por una revisión de la forma en la cual se ha incorporado la perspectiva de género en educación básica y media. Una tercera sección trata el currículum y su rol en la enseñanza en perspectiva de género, seguida por una cuarta sección donde se reseñan los distintos obstáculos para la incorporación de la perspectiva de género. Finalmente, se plantea una discusión acerca de los temas tratados. 


\section{ANTECEDENTES}

Para poder hablar de la perspectiva de género en educación con una visión amplia y contextualizada, deben abordarse primero ciertos antecedentes de la historia reciente que han acercado el trabajo de la perspectiva de género en el ámbito educativo.

En la introducción se planteó que el género es un aprendizaje sociocultural, por lo cual su percepción y vivir debe ser cambiado a través de nuevas prácticas culturales. Sin embargo, las prácticas culturales que afectan a la equidad de género, y al cambio social, corresponden a prácticas que tardan en ser cambiadas, puesto que se encuentran basadas en estructuras previamente definidas, que cumplen el rol de barreras ideológicas (Arcos et al., 2007). Por su parte, los procesos de socialización escolar influyen de manera directa en las pautas de comportamiento, en particular aquellas diferenciales por género. Debido a que dichas diferencias son las pautas hegemónicas de la sociedad, el sistema educativo tiende a reproducirlas, convirtiéndose la escuela en una instancia socializadora en la cual se refuerzan los privilegios de un género por sobre el otro (Rodríguez y Peña, 2005). En la cultura chilena, este género privilegiado por las estructuras de la sociedad corresponde al masculino, y es la eliminación del privilegio de un género por sobre otro a lo que apuntan las nuevas prácticas culturales que son resistidas (Arcos et al., 2007).

Buscando un origen a la relevancia de la perspectiva de género en el mundo, nos encontramos con la publicación del informe "Nuestra Diversidad Creativa" de la Comisión Mundial de Cultura y Desarrollo, ante la Organización de las Naciones Unidas para la Educación, Ciencia y Cultura (UNESCO por sus siglas en inglés). Este informe fue publicado el año 1996, y en él se plantea la cultura como clave para el desarrollo de los países, ilustrando su interacción con otros elementos, como la educación y la igualdad de género (Alonso y Medici, 2012). Posteriormente, la igualdad de género se convierte en un indicador del grado de desarrollo de los países (Colás y Jiménez, 2006). En consecuencia, comienza a dársele importancia al enfoque o perspectiva de género en distintos ámbitos, a nivel mundial. Como se mencionó en la introducción, la perspectiva de género busca el cambio de las expectativas tradicionales y estereotipadas de lo que es ser hombre (considerado lo dominante, y de la esfera pública) y ser mujer (considerado lo dominado, y de la esfera privada), reconociendo así la existencia de formas diferentes de ser hombres o mujeres (Gómez, 2009).

Teniendo en cuenta que la igualdad de género no es la norma o pauta dominante en ningún país (UNESCO, 2012), que las normas sociales afectan a la equidad de género (Cooray y Potrafke, 2011), y la incorporación de la Igualdad de Género a los Objetivos de Desarrollo Sostenible del Programa de las Naciones Unidas para el Desarrollo (PNUD) en el año 2016 (PNUD, 2018), los distintos gobiernos del mundo trabajaron en la incorporación de la promoción del principio de igualdad de género de manera explícita en sus políticas (Messina, 2001), esto, gracias al compromiso de estos gobiernos adheridos a las Naciones Unidas a cumplir con los acuerdos establecidos en relación a la transversalización de la perspectiva de género (Buquet, 2011). Sin embargo, la 
adopción de medidas que acerquen la perspectiva de género a la totalidad de las naciones no se ha dado de manera simultánea. Por ejemplo, a nivel latinoamericano, el énfasis en la igualdad de género surge en la década de 1980 en Costa Rica, planteándose leyes y programas que buscan la equidad entre hombres y mujeres (Solís Pérez, 2006). Esto contrasta con el caso chileno, donde el enfoque de género se incluye como uno de los aspectos estratégicos del Programa de Mejoramiento de la Gestión desde el año 2002 (Arcos et al., 2007), posterior al informe publicado ante la UNESCO.

En conjunto a la incorporación explícita de la perspectiva de género en los distintos gobiernos, comenzó a reconocerse que la conciencia de género compromete a todas las personas de la sociedad, incluyéndose en esto a educadores y administradores de la educación (Messina,2001), siendo el docente un ente activo que cuestiona la comprensión actual del género de niños y niñas (Blaise, 2009). Hasta entonces, género y educación solían ser consideradas esferas separadas, centrándose la investigación educativa en la determinación de diferencias de género entre niños y niñas (Rodríguez y Peña, 2005). El abordaje del tema es, entonces, el siguiente: para obtener cambio social, deben ser identificadas y transformadas las cosmovisiones valóricas y normativas de cada sociedad, incluyendo las de género. Dichas cosmovisiones surgen en la escuela, ya que la educación cumple el fin de velar por el desarrollo integral de individuos de una determinada cultura, transmitiendo cultura por medio del currículum explícito y la acción educativa (Gómez, 2009). La educación afecta el proceso de desarrollo de identidad de género de niños y niñas, proceso en el cual niños y niñas construyen discursos a través de lo que ven en el día a día (Blaise, 2009; Rodríguez y Peña, 2005). Por ello, la intervención a nivel educativo permite lidiar tempranamente con posibilidades y conflictos que hombres y mujeres tienen en su desarrollo personal y profesional, dándoles herramientas para problematizar y, consecuentemente, transformar su cultura (Arcos et al., 2007), evitando así la reproducción de pautas de acciones sociales que llevan a prácticas discriminatorias y sesgadas (Gómez, 2009). Por otro lado, el intervenir en la concientización sobre género en el ámbito educativo, permite que la información no quede solo en niños, niñas y docentes, si no que llegue a las familias de estos estudiantes y profesionales, beneficiando a un mayor número de individuos (Solís Pérez, 2006).

\section{INCORPORACIÓN DE PERSPECTIVA DE GÉNERO EN LA EDUCACIÓN}

En esta sección, se comentarán las formas en las cuales la perspectiva de género ha sido abordada en el ámbito educativo, con el fin de apreciar las distintas formas en las cuales este enfoque puede ser incorporado en la educación básica y media.

Al hablar de la perspectiva de género, puede uno preguntarse dónde podría apreciarse la misma en la educación. Ante esto, Messina (2001) enuncia que el género incide en más de un área educativa, por lo cual debe ser abordado con un enfoque holístico. En Latinoamérica, se implementan distintas políticas de igualdad de género en educación clasificadas por la autora en: 
- aquellas que mejoran el acceso;

- aquellas que mejoran el proceso educativo;

- aquellas que mejoran las posibilidades de egreso de la educación básica y media.

Por su parte, Colás y Jiménez (2006) identifican cuatro líneas de trabajo en la investigación sobre el género en educación, correspondientes a:

- la escolarización de acuerdo con el género;

- el currículum y su enfoque androcéntrico implícito;

- el aprendizaje de estudiantes de acuerdo con su sexo y las expectativas académicas de docentes;

- el profesorado, la transmisión de estereotipos y los modelos educativos que sustentan su formación inicial.

En conjunto, ambas clasificaciones abordan las distintas variables que afectan la problemática de manera directa e indirecta, puesto que niños y niñas presentan diferencias tanto en el acceso al sistema, como durante la experiencia escolar y en los resultados obtenidos.

Sin embargo, pese al gran número de posibles políticas en estas categorías, puede verse en diversos artículos que los primeros esfuerzos tendieron a enfocarse exclusivamente en asegurar un acceso igualitario a la educación, es decir, se enfocaron en el acceso de niñas a la escuela. Esto debido a que, hasta la década de 1990 en Latinoamérica, el acceso de niñas a la educación era desigual al de niños, lo cual explica por qué estos primeros esfuerzos en la igualdad de género en educación se dedican a asegurar el acceso igualitario. Por otro lado, estudios confirman que la escolaridad de mujeres conlleva a consecuencias positivas en el cuidado de su salud reproductiva, en la inserción laboral de la mujer (Messina, 2001), y en el aprendizaje y salud de sus hijos e hijas (Cooray y Potrafke, 2011). La escolaridad femenina tiene consecuencias en distintos ámbitos de la vida cotidiana, afectando incluso a las nuevas generaciones, lo cual justifica el énfasis en su acceso a la educación.

Avanzando los años, pese a la falta de datos desagregados por sexo en el total de los países latinoamericanos, se descubre que niñas participan en la educación básica casi tanto como los niños. Messina (2001) plantea que la desigualdad de acceso se da en casos de países que, de por sí, tienen altas tasas de analfabetismo, o altas tasas de población identificada como indígena. Las diferencias de acceso, continúa Messina (2001), se deben más a las diferencias socioeconómicas entre las familias de estudiantes, que al género de niños y niñas que acceden a la educación, indicándose que las diferencias en nivel de analfabetismo según género aumentan al integrar la variable de edad, sector rural, condición de pobreza y/o pertenencia a comunidades indígenas, no encontrándose el género como una causa aislada. La diferencia con relación a género solo se da en casos de extrema pobreza, quedando las mujeres de grupos vulnerables marginadas en la educación hasta el día de hoy, diferencia que se acentúa en la enseñanza media. El hecho que esta desigualdad de acceso persista durante la enseñanza media es preocupante, puesto que, de acuerdo con lo planteado por Arcos et al. (2007), se ve a este nivel 
educativo como el factor clave que incide, simultáneamente, en la equidad, el desarrollo integral y el ejercicio de la ciudadanía plena de los y las estudiantes.

Por el previo énfasis al acceso igualitario, el abordaje de cómo realizar la enseñanza con perspectiva de género ha tomado relevancia solo recientemente. Aquí nos encontramos con las políticas enfocadas en mejorar el proceso educativo, donde puede verse la incorporación del enfoque de género en la enseñanza misma. Sin embargo, el género se considera un tema de bajo interés, debido a la priorización que se tiene hacia los resultados obtenidos por los y las estudiantes en pruebas estandarizadas (Madrid, 2006). En el caso chileno, de haber preocupación en el enfoque de género, suele centrarse en contrastar los niveles de aprendizaje de niños y niñas (Arcos et al.,2007), debido a la demostrada constante estimulación positiva hacia niños por sobre las niñas, de parte del profesorado (Messina, 2001). Como consecuencia, se deja de lado el interés en las pautas de conducta en torno al género del profesorado, el currículum y el alumnado. Si bien los niveles de aprendizajes de niños y niñas son temas de preocupación, deben también abordarse las pautas mencionadas.

En conjunto a lo anterior, Arcos et al. (2007) enuncian que, en las contadas instancias en las cuales se ha implementado el enfoque de género en educación, se ha revelado que los programas educativos no satisfacen las necesidades específicas diferenciadas por género, llegando a reproducir y reforzar las desigualdades entre géneros, encontrándose en textos escolares la reproducción de aquellos estereotipos que ligan al hombre con lo cultural y a la mujer con lo natural (Viotti y Del Valle, 2013). Así, entonces, se privilegian valores androcéntricos en los programas educativos, sin cuestionamientos del rol que se le asigna a priori a hombres y mujeres en función de su género.

\section{EL ROL DEL CURRÍCULUM EN LA EDUCACIÓN}

Para continuar con las ideas anteriormente planteadas, se desarrollará en esta sección la revisión de +temáticas de currículum educativo, y su relevancia en la pedagogía en igualdad de género. El término currículum surge en el sistema educativo chileno a fines del siglo XIX, y puede ser tradicionalmente definido de la siguiente manera:

Prescripción obligatoria para todo el sistema escolar, de un plan de estudios, o definición de áreas de conocimiento, tiempos de trabajo asociados y su secuencia en el total de la experiencia de la escolaridad, y un programa de estudios, o los objetivos de aprendizaje, contenidos y orientaciones didácticas para el docente organizados por unidades en cada año escolar. (Cox, 2011, p. 2)

En aquella definición, se consideran dos elementos fundamentales, correspondientes al plan de estudio y al programa de estudios. Actualmente, existe una distinción entre marco curricular, definido por el Ministerio de Educación, y los planes y programas de estudio, que quedan a criterio de cada establecimiento escolar, teniendo estos la opción de elaborar planes propios o aplicar aquellos 
previamente definidos por el Ministerio de Educación (Cox, 2011). Esto genera que un mismo tema pueda verse abordado de maneras muy diferentes, de acuerdo con el interés propio de cada establecimiento educacional, siempre que siga los lineamientos básicos del marco curricular.

Paralelamente, el currículum es visto como la concreción de un proyecto social y cultural, formulado en términos de intervenciones educativas y medios para obtenerla (Coll, 2013), representando así el conocimiento seleccionado y organizado de manera legítima para su enseñanza y aprendizaje. De esta forma, los currículums escolares son una acción a través de la cual se pueden transmitir las ideologías elegidas por quienes los estructuran (Magendzo, 2002). Con lo anterior en consideración, debe reconocerse que, en educación, se considera la existencia de cuatro currículums simultáneamente en ejecución, correspondientes al formal, el en acción, el obviado y el oculto (Arcos et al., 2007). Más allá de la transmisión de estereotipos sexistas en la enseñanza a través de textos y manuales escolares, el género no solía encontrarse en el currículum formal ni en acción (Messina, 2001). Por su parte, el currículum oculto juega un rol fundamental en la configuración de significados y valores de manera inconsciente, mientras que el obviado facilita el ignorar interrogantes sobre temas transversales (Arcos et al., 2007). Los y las docentes, no cuentan con una guía establecida de cómo tratar temáticas de género, puesto que es un tema que no se encuentra explicitado dentro del currículum formal. Por ello, los temas relacionados al género se trabajan desde el currículum obviado y el oculto, impartiendo los docentes (de forma inconsciente) aquello que se alinea con sus creencias personales, cayendo en prácticas sexistas en más de una ocasión.

Lo anterior, conlleva a que las ideas de igualdad de género, roles de género y estereotipos tienden a ser impartidas de manera inconsciente, no planteándose las interrogantes ni las necesidades del alumnado en temáticas de género. Considerando que la identidad de género se construye a través de la interacción entre sujetos en distintos contextos escolares, por medio de discursos y otras formas de comunicación (Blaise, 2009; Colás y Jiménez, 2006), el discurso inconsciente y no regulado del profesorado tiene fuertes repercusiones en la formación de sus estudiantes como personas (Colás y Jiménez, 2006). Es más, un estudio español detecta que la forma de pensar de los docentes de la actualidad no ha variado de la forma de pensar propia de fines del siglo $\mathrm{XX}$, perdurando los prejuicios relativos al sistema sexo-género, donde se le asignan distintas habilidades, cualidades y expectativas, a hombres y mujeres, en base a su sexo biológico (Díaz de Greñu y Anguita, 2017). Esto concuerda con lo planteado por Rebollo et al. (2011), quienes indican que el profesorado suele no ser consciente de cómo sus creencias de género particulares afectan su docencia, sin percatarse de las prácticas sexistas a las cuales incurren por omisión. Es parte de su forma de pensar, y los hace actuar de tal manera que la desigualdad y discriminación entre niños y niñas dentro del sistema educativo ya no se encuentra manifiesta de manera explícita, pero persiste en el currículum oculto, por medio de "decisiones en los contenidos, estrategias metodológicas, relaciones interpersonales, elección de recursos didácticos, distribución del espacio y asignación de tareas" (Díaz de Greñu 
y Anguita, 2017, p. 220), decisiones generalmente tomadas por el profesorado. Entre las prácticas provenientes del currículum oculto mencionadas se incluyen, por ejemplo, la existencia de un trato diferencial del profesorado hacia niños o niñas (Romero y Abril, 2008).

El género, por lo tanto, debiera encontrarse explicitado en el currículum, para prevenir que se presenten los discursos sesgados como aquellos más disponibles y accesibles al estudiantado (Blaise, 2009; Rodríguez y Peña, 2005). Esto es vital para evitar una comprensión errónea y sesgada del mundo social, por parte de niños y niñas en formación. Después de todo, un currículum que ignora la diversidad de temas nuevos y renovados, de continua emergencia, sin reconocerlos o integrarlos al conocimiento disciplinario, no responde a las necesidades de la realidad, convirtiéndose en obsoleto, impertinente e irrelevante (Magendzo, 2002).

En la actualidad, se considera que el sistema educativo tiene la responsabilidad de combatir, a través de la implementación de nuevas prácticas educativas, la falta de equidad entre hombres y mujeres (Suberviola, 2012), enfrentando así el desafío de la liberación de estereotipos de género (Díaz de Greñu y Anguita, 2017). Estos estereotipos son transmitidos por el profesorado a sus estudiantes, influyendo y afectando de manera directa los programas escolares, las interacciones en el aula y el respeto a la intimidad de niños y niñas; llevando a la persistencia de la discriminación de género y a su perpetuación en el sistema educativo (Colom y Mayoral, 2016; Díaz de Greñu y Anguita, 2017; Suberviola, 2012). Tal como se ha recalcado anteriormente, el sistema educativo es el contexto idóneo para facilitar avances en la igualdad de oportunidades entre hombres y mujeres (Suberviola, 2012), y para crear espacios abiertos a la reflexión, donde se les permita a estudiantes generar una conciencia crítica, con la guía del docente ya concientizado (Fueyo y Fernández Del Castro, 2012).

En el ámbito de la enseñanza misma, debe hablarse de una pedagogía de género, donde es necesaria la elaboración de propuestas de género que permita al profesorado fomentar la igualdad entre personas en general, y estudiantes en particular, sin generar distinciones en base al género del individuo (Solís Pérez, 2006), trabajándose la denominada "conciencia de género" (Messina, 2001). La institución educativa tiene la capacidad de influir en el desarrollo de identidades de género flexibles y abiertas, por medio de la acción docente (Rodríguez, 2007), cumpliendo así un rol de "agente activo" en la transformación de pautas sociales (Rebollo et al., 2011). Esto es solo posible si se dispone de un currículum que dé el espacio a efectuar dichas acciones.

En este contexto, en Latinoamérica se ha incorporado la perspectiva de género en el currículum explícito, en materiales y manuales para la producción de material de enseñanza no sexista, en la educación sexual, y en los programas de formación continua para docentes (Messina, 2001), buscando disminuir con los estereotipos sexistas en libros de texto y el enfoque androcéntrico de los mismos (Anguita, 2011; Romero y Abril, 2008). También se busca disminuir el trato diferencial del profesorado hacia los estudiantes masculinos por sobre las estudiantes femeninas (Romero y Abril,2008). Aun así, este trabajo es incipiente, por lo cual queda mucho que hacer en esta área. 


\section{LOS OBSTÁCULOS PARA UNA EDUCACIÓN CON ENFOQUE DE GÉNERO}

Existen múltiples barreras para la aplicación de un enfoque de género. A continuación, se enuncian algunos de los obstáculos planteados en la bibliografía, con un breve desarrollo de cada uno de ellos, para dar una visión de la problemática abordada.

\section{RÉGIMEN DE GÉNERO PROPIO DE CADA ESTABLECIMIENTO EDUCACIONAL}

Rebollo et al. (2011) plantean que las ideologías en general, y la ideología de género en particular, no surgen de manera espontánea, sino que existen previamente en las instituciones de la sociedad. Los autores enuncian que, a través de estas instituciones, dentro de las cuales se incluyen los establecimientos educacionales de enseñanza básica y media, se transmiten las prácticas y políticas vinculadas a las ideologías. Las instituciones, por lo tanto, cuentan con "coordenadas culturales" específicas, que influyen en su forma de ver y entender diversos fenómenos. En el caso particular de los establecimientos educacionales, estos producen y reproducen modelos (Madrid, 2006), cumpliendo la función de transmisión de cultura por medio del currículum explícito y la acción educativa desarrollada en la cultura escolar.

Por todo lo anterior, de acuerdo con Rodríguez y Peña (2005), cada establecimiento educacional cuenta con su propio régimen de género, puesto que este corresponde a un tema intrínseco de la formación de niños y niñas. Los regímenes se conforman por expectativas, reglas, rutinas, códigos y por el orden jerárquico establecido, lo cual lleva a la creación de distintos "repertorios" de acción, insertados en el currículum oculto de cada establecimiento (Madrid, 2006; Rodríguez y Peña, 2005). Dichos repertorios, generan profundos efectos en las condiciones en las que niños y niñas configuran su identidad personal, incluyendo su identidad de género. En conjunto a esto, existe una "inercia institucional", correspondiente a las costumbres y hábitos ya existentes en los establecimientos educacionales (Rebollo et al., 2011), desde la que se tiende a rechazar los cambios y las nuevas perspectivas, lo cual tiene efectos negativos en los procesos de cambio que se lleven a cabo en el ambiente escolar, entre los que se incluye la incorporación de una nueva forma de ver la perspectiva de género. Esta inercia forma parte de los factores clave para comprender la resistencia de centros educativos a incorporar innovaciones con relación al género, postulados por Rebollo et al. (2011), en conjunto a la ideología de género (explícita o implícita) de cada establecimiento.

Lo planteado se ve tanto en los establecimientos de educación básica y media, como en los establecimientos de educación superior, donde el tratamiento de temas como el género, la no discriminación, la tolerancia, la formación ciudadana, la sexualidad y la convivencia escolar, dependen del proyecto educativo de cada institución (Madrid, 2006). Esto es de gran importancia, si se considera que un tratamiento pobre de temáticas de género y sexualidad lleva a la reproducción de pautas de acciones sociales discriminatorias y sesgadas que no tienen perspectiva de género (Gómez, 2009). Por ello, es de alto impacto el régimen de género 
propio de cada establecimiento educacional, en particular si se considera que el trato de estos temas será distinto entre un establecimiento educacional y otro. Es un punto a trabajar si se quiere llegar a la incorporación de una perspectiva de género homogénea a nivel nacional, independiente del régimen propio de cada establecimiento.

\section{FALTA DE PRIORIZACIÓN Y VISIBILIZACIÓN DE LA DISCRIMINACIÓN DE GÉNERO, Y FALTA DE MODELOS Y POLÍTICAS PÚBLICAS REALISTAS DE ACCIÓN}

En este punto se aborda el obstáculo correspondiente a la falta de priorización, visibilización y no abordaje en el ámbito educativo de la discriminación de género, con pocas propuestas realistas de modelos, estrategias educativas, materiales, herramientas y métodos para trabajar el tema.

La principal arista de este obstáculo corresponde a que la discriminación de género se considera un tema no prioritario, puesto que ya se ve como superado (Madrid,2006; Romero y Abril, 2008), pese a no ser así. Lo anterior conlleva a que no sea un tema abordado en el ámbito educativo (Gómez, 2009), a que falte investigación empírica cuantitativa y cualitativa, transversal y longitudinal (Madrid, 2006), y a que, debido a ello, no haya materiales, métodos, herramientas ni claridad sobre cómo tratar temáticas de género en la enseñanza (Gómez, 2009; Romero y Abril, 2008). El sistema educativo continúa dirigiéndose, principalmente, hacia la escolarización universal, ignorando las problemáticas de la actualidad (Coll, 2013). A causa de esto, de acuerdo con Gómez (2009), las escuelas, no contarían con las herramientas, los conocimientos o las actitudes requeridos para responder a las necesidades de formación del alumnado. Las acciones que durante los años '90 se encontraban dirigidas a la igualdad de género, tenían un lugar secundario en la política educativa, no llegando a constituir un cuerpo coherente (Madrid, 2006). Por su parte, el Estado ignora y omite el tema, no implementando políticas públicas para tratar con el enfoque de género. La combinación del proceso omisión del Estado y la ausencia de herramientas en los establecimientos educacionales se convierte en la piedra angular de la falta de respuesta a las necesidades de formación básicas del alumnado.

En los casos donde se incorpora la perspectiva de género en los currículums explícitos y en la práctica educativa, se detecta que el material de enseñanza aún transmite estereotipos sexistas, omitiendo también los aportes realizados por mujeres en campos científicos, históricos y sociales, por lo cual es necesario continuar el trabajo en el trato del currículum oculto (Anguita, 2011; Madrid, 2006; Messina, 2001; Romero y Abril, 2008). Falta, también, la visibilización de la discriminación de género en contenidos, metodologías y prácticas de enseñanza (Colás y Jiménez, 2006). La situación, por supuesto, es peor en aquellos establecimientos donde aún no se hace la incorporación de la perspectiva de género en el currículum explícito.

Por otro lado, las políticas públicas que sí se encuentran implementadas, tienen un abordaje alejado de la realidad, debido a la falta de investigación empírica anteriormente mencionada (Gómez, 2009; Madrid, 2006). Así, las políticas 
públicas existentes fallan en su discurso al analizar la realidad correctamente, sin acoplarse a la misma (Gómez, 2009). Viene a existir un vacío en política de género que atienda las Necesidades Especiales de niños, niñas y docentes (Madrid, 2006), persistiendo así las conductas discriminatorias y sexistas en el aula, por lo cual es necesaria la implementación de políticas públicas que aborden el género desde un punto de vista más cercano a la realidad.

\section{RESISTENCIA DESDE AQUELLOS SECTORES MÁS CONSERVADORES DE LA SOCIEDAD}

Un tercer obstáculo corresponde a la resistencia a la incorporación de la perspectiva de género en la educación básica y media, de parte de aquellos sectores más conservadores de la sociedad. Esta resistencia, ejercida a través de presión social, impide que buenas prácticas o iniciativas se conviertan en políticas públicas del sistema educativo (Madrid, 2006; Messina, 2001).

La influencia que tienen hasta el día de hoy los grupos más conservadores puede entenderse como parte de la caracterización patriarcal de la sociedad, donde se considera la supremacía innata de los hombres por sobre las mujeres, utilizándose justificaciones tanto religiosas como científicas y del lenguaje (Solís Pérez, 2006). Por otro lado, de acuerdo con Madrid (2006), el tema es aún incipiente en Latinoamérica, estando más presente en países desarrollados. De momento, continúa el autor, en Latinoamérica se prioriza el acceso a la educación por sobre las relaciones de género y el cuestionamiento de los patrones de dominación que persisten en la sociedad, es decir, no se cuestiona el rol de estos grupos conservadores.

Por otro lado, en la formulación de currículums escolares, interviene una serie de actores sociales, cada uno con una posición ideológica propia, causando que los conocimientos seleccionados representen la visión particular de quienes producen el conocimiento, y de quienes lo reordenan en el currículum (Magendzo, 2002). Por ello, en lugares con mayor influencia de los sectores conservadores de la sociedad, se verá una predominancia de dichas ideologías en las enseñanzas del sistema educativo. Esto se vincula directamente con el primer obstáculo reseñado, correspondiente a la existencia de regímenes de género propios en cada establecimiento educacional.

\section{NO INSTITUCIONALIZACIÓN DE LA PERSPECTIVA DE GÉNERO EN EL PROFESORADO}

Si bien está demostrado que tanto docentes como administrativos y estudiantes perciben diferencias estereotipadas entre hombres y mujeres en las instancias de enseñanza (Solís Pérez, 2006), la integración de la perspectiva de género en la formación docente se queda en un par de experiencias puntuales, casi anecdóticas, sin ir más allá (Anguita, 2011; Messina, 2001). La formación docente se encuentra al debe, puesto que no existe una política en este ámbito, si no que un par de acciones innovadoras aisladas (Madrid, 2006), posiblemente por la invisibilización de la problemática (Anguita, 2011). Por ello, plantean Romero y Abril (2008), cuando docentes u otros profesionales de la educación trabajan temáticas de género, es debido a una iniciativa individual, considerando 
el interés o la sensibilidad propios de un docente determinado, dándose casos de profesionales de la educación que no consideran necesarias las competencias de género, puesto que no son una prioridad en su forma de enseñar.

Lo anterior lleva a concluir como necesario mejorar los aspectos relacionados al género en la formación del profesorado (Rebollo et al.,2011), puesto que es un tema central en la incorporación de una perspectiva de género en la educación (Madrid, 2006), siendo también necesario el sensibilizar a los y las profesionales, para que sus lecciones sean, efectivamente, planificadas desde una perspectiva de género (Solís Pérez, 2006). Actualmente, Madrid (2006) plantea que, en la formación inicial docente, los ramos de educación y género suelen ser de carácter optativo, es decir, sólo son tomados por aquellas personas previamente interesadas; o de carácter transversal, donde el tema no se aborda explícitamente. Romero y Abril (2008) añaden que las temáticas de género suelen verse en asignaturas más teóricas que didácticas en las Universidades, dificultando la implementación de técnicas didácticas en la enseñanza de los y las futuras profesoras.

En cuanto a la formación continua, se ha visto que los docentes desconfían de tratar el enfoque de género, puesto que sienten cuestionadas sus prácticas docentes previas (Romero y Abril, 2008). Lo que se requiere, entonces, es la creación e implementación de nuevos modelos pedagógicos, con una formación del profesorado vinculada estrechamente a la perspectiva de género, que le permita crear una conciencia crítica en temáticas asociadas (Anguita, 2011; Colás y Jiménez, 2006), tomando en consideración las creencias y opiniones de quienes actualmente practican la docencia, puesto que su cambio de mentalidad es clave para la realización de una intervención adecuada y eficaz en el sistema educativo (Rodríguez, 2007).

\section{DESACUERDO EN CÓMO INCORPORAR EL ENFOQUE DE GÉNERO EN PLANES DE ESTUDIO}

Actualmente, la forma principal de la incorporación del enfoque de género en planes de estudio, de acuerdo con Messina (2001), es la integración de manera transversal, es decir, la incorporación de contenidos de género en las disciplinas ya enseñadas. Este enfoque tiene sus ventajas y desventajas. Como ventaja, se encuentra el hecho que, al ser abordado de manera transversal, el tema se encuentra reforzado en el total de asignaturas. Como desventaja, esto puede conllevar a la invisibilización del tema, al no abordarse en un espacio dedicado y explícito (Madrid, 2006), realizando así un abordaje de manera tangencial y poco significativa de los problemas de las mujeres en el mundo (Fueyo y Fernández Del Castro, 2012). Esto suele generar un desacuerdo entre las personas encargadas de crear los planes de estudio, causando que las acciones y prácticas dirigidas al establecimiento de la equidad de género en el ámbito educativo escolar hayan tenido un carácter parcial, interrumpido y no siempre coherente (Madrid, 2006).

Paralelamente, de acuerdo con Colás y Jiménez (2006), existen tres enfoques teóricos para explicar el género en el contexto educativo, correspondientes a las teorías feministas, la pedagogía crítica y el enfoque sociocultural, los cuales 
los autores explican de la siguiente manera: Las teorías feministas buscan realizar transformaciones que posibiliten las relaciones de género en equidad, aportando a la pedagogía con una perspectiva social que permite estudiar, intervenir y explicar los procesos educativos, viendo qué se enseña, cómo se enseña y cómo se aprende lo enseñado. La pedagogía crítica, por su parte, articula la pedagogía con la transformación social, considerándose la concientización como un proceso progresivo; a su vez, se considera que la "conciencia de género" es un filtro que permite la interpretación del mundo, siendo fundamental la educación en género durante su formación. Finalmente, el enfoque sociocultural da propuestas de metodologías que permiten la observación y reorientación de prácticas escolares, destacándose que la identidad de género se construye a través de la interacción entre sujetos en diversos contextos sociales, donde se incluye el contexto escolar. La incorporación de estas tres perspectivas al idear un plan de estudio sería lo ideal, pero, sin embargo, en la práctica suele considerarse un enfoque por sobre los otros, o, peor aún, ninguno de los anteriores.

\section{DISCUSIÓN}

En base a todo lo anterior, está claro que la escuela fomenta actitudes y experiencias en niños y niñas desde temprana edad, siendo un agente activo en su formación identitaria. Esto la convierte en el lugar perfecto para incentivar la eliminación de estereotipos sexistas y otros indicadores de la desigualdad entre géneros. Sin embargo, pese a que la escuela como Institución es el agente principal, debe considerarse que interactúa tanto con su propio currículum, como con la formación del profesorado que trabaja en él.

Estos tres elementos se articulan como factores clave en la reformulación de la educación desde y para la equidad de género (Colás y Jiménez, 2006). Por ende, está justificada la necesidad de considerar la igualdad de género en la elaboración de programas, planes de estudio y otros materiales educativos, como lo son los textos escolares, además de considerarla al realizar actividades dinámicas en el aula. También debe ser considerada en la práctica docente y en la misión y visión de los establecimientos educacionales.

En la actualidad, las diferencias formales y explícitas, que existían con relación al género en las escuelas, están desapareciendo. No obstante, se mantienen los mecanismos y procesos implícitos que refuerzan los estereotipos de género (Rodríguez y Peña, 2005), por lo cual debe continuarse el trabajo de la incorporación de la perspectiva de género en los planes de estudio, y en las políticas escolares, tanto en el currículum explícito como en el oculto, es decir, en las prácticas docentes. Ante esto, Suberviola (2012) propone tres principios para el desarrollo de planes para la igualdad entre hombres y mujeres, que corresponden a la visibilidad, la transversalidad y la inclusión. La visibilidad corresponde a mostrar diferencias entre sexos, con el fin de reconocer las desigualdades y discriminaciones generadas a partir de ellas; la transversalidad, tal como planteaba Messina (2001), refiere a encontrar la perspectiva de género en el conjunto de acciones y planes; y la inclusión refiere a realizar intervenciones en niños y niñas, para corregir las 
desigualdades generadas por los papeles tradicionales estereotipados de acuerdo al género, con el fin de conciliar intereses y crear relaciones de género igualitarias. Coll (2013), plantea que, para mejorar el aprendizaje de estudiantes en distintos ámbitos, debe considerarse el contexto particular de cada uno de ellos, más allá de la educación formal, relevando la importancia de las denominadas trayectorias individuales de aprendizaje. Incorporar este enfoque al modo en que se enseña la perspectiva de género puede tener consecuencias positivas en niños y niñas, quienes incorporarán no solo lo visto en la escuela, si no que aquello visto en el día a día de sus hogares, realizándose una enseñanza más participativa.

Considerando estas perspectivas de manera conjunta, surge la necesidad de espacios y tiempos escolares "abiertos a la reflexión", donde se les permita a estudiantes generar pensamientos convergentes y divergentes sobre el género (Fueyo y Fernández Del Castro, 2012). Esto requiere que el tema sea abordado de manera explícita en el ambiente escolar, además de transversal, dando a estudiantes y docentes la oportunidad de dialogar en temáticas de género, trabajando así las verdaderas necesidades del alumnado. Es así que el trabajo debe realizarse no sólo con alumnos, si no que con directivos, profesores y familias de los alumnos en los distintos establecimientos educacionales (Gómez, 2009), diseñando programas que afronten las desigualdades históricas de distintos agentes de la sociedad, en busca de equilibrar a las poblaciones que se han mantenido en desventaja a través de los años (Buquet, 2011), como lo es el caso de la población femenina. La reflexión debe realizarse en todos los estamentos que forman parte de la enseñanza básica y media, para evitar que estudiantes se encuentren con discursos contradictorios y discriminatorios.

Pese a que ya se destacó la necesidad del trabajo en la totalidad de los estamentos, debe recalcarse que lo fundamental corresponde a la guía del docente previamente sensibilizado en temas vinculados a la igualdad de género, $y$ concientizado en la necesidad de erradicar las prácticas discriminatorias en el aula (Solís Pérez, 2006). Por ello, si bien el currículum tiene un rol fundamental en orientar cómo realizar la enseñanza, no puede descartarse la necesidad de nuevos modelos pedagógicos, y una formación del profesorado específica (Colás y Jiménez, 2006), donde se considere la perspectiva de género. Por ello, durante la formación del profesorado, debe permitirse la creación de una conciencia crítica del mismo en cuestiones de género, lo cual es clave para la sensibilización de docentes. Esto permitirá la generación de aquellas innovaciones y transformaciones educativas necesarias (Colás y Jiménez, 2006; Rebollo et al., 2011), incluyendo aquellas del currículum explícito y obviado. Esto evitará que el currículum oculto manifieste puntos de vista sesgados y que perpetúen los estereotipos de género.

Debido a lo anterior, debe trabajarse la perspectiva de género tanto en la formación inicial como continua de quienes ejercen y ejercerán la docencia, es decir, no sólo debe estar considerado en el currículum escolar, sino que también en los planes de estudio de la Educación Superior, y en las planificaciones de los cursos de formación continua. Después de todo, debe considerarse que, de acuerdo a Madrid (2006), las políticas que funcionan son aquellas que intervienen en 
la escuela como un todo, generando docentes sensibilizados y capacitados, que toman la experiencia de alumnos como punto central del aprendizaje. Los otros dos factores clave para Madrid corresponden a la continuidad y coherencia en el tiempo de la política, y, finalmente, la explicitación de las temáticas de género en el currículum.

Las estrategias previamente abordadas, indican que la escuela, el currículum y la formación del profesorado son los elementos clave para reformular la educación, para y desde la equidad de género (Colás y Jiménez, 2006). Así, estos tres elementos pueden trabajarse con la incorporación de nuevos contenidos, diversas estrategias y distintas perspectivas, con el fin de mejorar la educación de las futuras generaciones de estudiantes y docentes.

\section{REFERENCIAS}

ALONSO, G.; MEDICI, M. Batería de indicadores UNESCO en cultura para el desarrollo: Una herramienta para integrar la cultura en las estrategias de desarrollo. Cultura y Desarrollo, Habana, n. 7, p. 12-17, 2012.

ANGUITA, R. El reto de la formación del profesorado para la igualdad. Revista Electrónica Interuniversitaria de Formación de Profesorado, Zaragoza, v. 14, n. 1, p. 43-51, abr. 2011.

ARCOS, E. et al. Estado del arte y fundamentos para la construcción de indicadores de género en educación. Estudios Pedagógicos, Valdivia, v. 33, n. 2, p. 121-130, dic. 2007. http://doi.org/10.4067/S0718-07052007000200007

BLAISE, M. "What a girl wants, what a girl needs": Responding to sex, gender and sexuality in the early childhood classrooms. Journal of Research in Childhood Education, Washington, D.C., v. 23, n. 4, p. 450-460, nov. 2009. https://doi. org/10.1080/02568540909594673

BUQUET, A. Transversalización de la perspectiva de género en la educación superior. Problemas conceptuales y prácticos. Perfiles Educativos, Ciudad de México, v. 33, n. esp., p. 211-225, ene. 2011.

COLÁS, P.; JIMÉNEZ, R. Tipos de conciencia de género del profesorado en los contextos escolares. Revista de Educación, Madrid, n. 340, p. 415-444, mayo/ago. 2006. COLL, C. El currículo escolar en el marco de la nueva ecología del aprendizaje. Aula de Innovación Educativa, Barcelona, n. 219, p. 31-36, feb. 2013.

COLOM, J.; MAYORAL, D. Participación, familia y escuela: la ausencia de lo femenino. Revista Electrónica Interuniversitaria de Formación del Profesorado, Zaragoza, v. 19, n. 1, p. 113-125, ene. 2016.

COORAY, A.; POTRAFKE, N. Gender inequality in education: political institutions or culture and religion? European Journal of Political Economy, Amsterdam, v. 27, n. 2, p. 268-280, jun. 2011. https://doi.org/10.1016/j.ejpoleco.2010.08.004

COX, C. Currículo escolar de Chile: génesis, implementación y desarrollo. Revue International d'Éducation de Sèvres, Sèvres, n. 56, p. 1-9, abr. 2011. 
DÍAZ DE GREÑU, S.; ANGUITA, R. Estereotipos del profesorado en torno al género y a la orientación sexual. Revista Electrónica Interuniversitaria de Formación de Profesorado, Zaragoza, v. 20, n. 1, p. 219-232, ene. 2017. https://doi. org/10.6018/reifop/20.1.228961

FUEYO, A.; FERNÁNDEZ DEL CASTRO, J. Hacer visible lo cotidiano a través del cine: la perspectiva de género en la educación para el desarrollo. Revista Electrónica Interuniversitaria de Formación del Profesorado, Zaragoza, v. 15, n. 2, p. 123-130, ago. 2012.

GÓMEZ, H. La pertinencia de una educación en sexualidad con perspectiva de género. Revista al Sur de Todo, Santiago, n. 1, p. 19-24, ene. 2009.

LAMAS, M. La antropología feminista y la categoría "género". Nueva Antropología, Ciudad de México, v. 8, n. 30, p. 173-198, nov. 1986.

MADRID, S. Profesorado, política educativa y género en Chile: balance y propuestas. Colección Ideas, Santiago, año 8, n. 76, p. 1-25, jun. 2006.

MAGENDZO, A. Derechos humanos y currículum escolar. Revista Instituto Interamericano de Derechos Humanos, San José, v. 36, p. 327-338, jul.-dic. 2002.

MESSINA, G. Estado del arte de la igualdad de género en la educación básica de América Latina (1990-2000). Santiago: Andros Impresores, 2001.

ORGANIZACIÓN DE LAS NACIONES UNIDAS PARA LA EDUCACIÓN, LA CIENCIA Y LA CULTURA (UNESCO). Indicadores UNESCO de cultura para el desarrollo: manual metodológico. París: Organización de las Naciones Unidas para la Educación, la Ciencia y la Cultura, 2012.

PROGRAMA DE LAS NACIONES UNIDAS PARA EL DESARROLLO (PNUD). Objetivos de desarrollo sostenible. PNUD, 2018. Disponible en: http:// www.undp.org/content/undp/es/home/sustainable-development-goals.html. Acceso en el: 20 sep. 2019.

REBOLLO, M. et al. Diagnóstico de la cultura de género en educación: actitudes del profesorado hacia la igualdad. Revista de Educación, Madrid, n. 355, p. 521-546, mayo/ago. 2011. http://doi.org/10.4438/1988.592X.RE.2010.355.035

RODRÍGUEZ, M. Opiniones de las maestras de Educación Infantil sobre el papel de la familia y la escuela en la configuración de la identidad de género. Revista Electrónica Interuniversitaria de Formación del Profesorado, Zaragoza, v. 10, n. 1, p. 1-9, ago. 2007.

RODRÍGUEZ, M.; PEÑA, J. La investigación sobre el género en la escuela: nuevas perspectivas teóricas. Teoría de la Educación, Salamanca, v. 17, p. 25-48, 2005.

ROMERO, A.; ABRIL, P. Género y la formación del profesorado en los estudios de educación infantil. Revista Electrónica Interuniversitaria de Formación del Profesorado, Zaragoza, v. 11, n. 3, p. 40-50, dic. 2008.

SOLÍS PÉREZ, Y. Propuesta metodológica para fomentar la igualdad de género en el aula escolar. Revista Electrónica Educare, Heredia, v. 10, n. 1, p. 103-122, nov. 2006. https://doi.org/10.15359/ree.10-1.6 
SUBERVIOLA, I. Coeducación: un derecho y un deber del profesorado. Revista Electrónica Interuniversitaria de Formación del Profesorado, Zaragoza, v. 15, n. 3, p. 59-67, oct. 2012.

VIOTTI, M.; DEL VALLE, A. Identidades de género en la escuela: una mirada desde el nivel inicial y el primer ciclo. La Alijaba, Segunda Época, Luján, v. 17, p. 169-178, oct. 2013.

\section{SOBRE LOS AUTORES}

Amanda Valenzuela-Valenzuela es magíster en lingüística aplicada por la Universidad de Concepción (Chile). Investigadora en la Fundación Kimntrum (Chile).

E-mail: amandavalenzuela93@gmail.com

Ricardo Cartes-Velásquez es doctor en ciencias médicas por la Universidad de La Frontera (Chile). Profesor en la Universidad Andrés Bello (Chile). E-mail: rcartesv@kimntrum.org 